\title{
Caracterización bromatológica de insumos no tradicionales para alimentación animal en la región Amazonas
}

\section{Bromatological characterization of non-traditional supplies for animal feed in the Amazonas region}

Wilmer Bernal', Jorge L. Maicelo² e Ives Yoplac ${ }^{3}$

RESUMER

El presente estudio se llevó a cabo en el Instituto de Investigación en Ganadería y Biotecnología de la UNTRM, con el objetivo de realizar la caracterización bromatológica de veinte insumos alimenticios disponibles en la región y puedan ser utilizados en alimentación de animales domésticos. La determinación de la composición química se realizó mediante análisis proximal, a fin de determinar materia seca (MS), proteína cruda (PC), fibra cruda (FC), extracto etéreo (EE), cenizas (CEN) y extracto libre de nitrógeno (ELN). Además, se determinó la concentración de minerales $\mathrm{Ca}, \mathrm{P}$, energía bruta (EB) y en insumos con aptitud para alimentación de rumiantes, se determinó fibra detergente neutro (FDN) y fibra detergente ácido (FDA). Los insumos se han clasificado de acuerdo a su naturaleza en cereales (maíz), raíces y tubérculos (harina de yuca, papa y bitucón), frutales (harinas de plátano, pulpa de naranja y guayaba) y subproductos agroindustriales (pulpa de café, cáscara de cacao, polvillo de arroz, nielen y arrocillo). La clasificación final de dichos insumos se realizó por la concentración de nutrientes, en proteicos, energéticos y fibrosos. Se concluye que en la región Amazonas, existe alto potencial de insumos para ser utilizados en la alimentación animal, tanto en monogástricos y rumiantes; sin embargo, es necesario continuar con la caracterización del perfil de aminoácidos y concentración de componentes antinutricionales en los insumos identificados.

\section{Palabras clave}

análisis proximal · caracterización nutricional insumos no tradicionales $\cdot$ subproducto

\section{INTRODUCCIÓN}

El alimento balanceado representa entre el 65 y $70 \%$ del costo de producción en las especies de interés zootécnico. La demanda actual de maíz amarillo no puede ser cubierta en su totalidad por el mercado
ABSTRACT

The present study was carried out at the Institute of Research in Livestock and Biotechnology of the UNTRM, with the objective of characterizing bromatology of 20 food supplies available in the region, and to be used in domestic animal feeding. The determination of the chemical composition was done by means of proximal analysis, to determine dry matter (DM), crude protein (CP), crude fiber (CF), ethereal extract (EE), ash (A) and nitrogen free extract (NFE). In addition, the concentration of minerals $\mathrm{Ca}, \mathrm{P}$, gross energy (GE) was determined; and in those inputs with ruminant feeding ability, neutral detergent fiber (NDF) and acid detergent fiber (ADF) were determined. Inputs have been classified according to their nature in: cereals (corn), roots and tubers (cassava flour, potato and bitucón), fruit trees (banana meal, orange pulp, guava), and agroindustry byproducts (coffee pulp, cocoa shell, rice dust, nielen and arrocillo). The final classification of these inputs was made by the concentration of nutrients in these inputs, in protein, energy and fibrous. It concludes that in the region, there is a high potential of inputs to be used in animal feed, monogastric and ruminants; however, it is necessary to continue the characterization of the amino acid profile and concentration of antinutritional components in the identified inputs.

\section{KEYWORDS}

proximate analysis · nutritional characterization nontraditional ingredients $\cdot$ by-products

local. Esto se acentúa con la disminución en un $10 \%$ de la producción nacional en el último año, por lo que depende de las importaciones. Esta dependencia es de hasta el 50 \%. En la actualidad, los principales países de donde se importa son Estados Uni-

\footnotetext{
'Ingeniero Zootecnista. Investigador del IGBI y docente FIZAB-UNTRM. Correo electrónico: wilmer.bernal@untrm.edu.pe ${ }^{2}$ Ingeniero Zootecnista. Investigador del IGBI y docente FIZAB-UNTRM. Correo electrónico: jmaicelo@untrm.edu.pe

${ }^{3}$ Ingeniero Agroindustrial. Investigador del IGBI y docente FIZAB-UNTRM. Correo electrónico: iyoplac.fizab@untrm.edu.pe
} 
dos (73\%) y Argentina (25\%) (Del Águila, 2016).

En el Perú, los cultivos de mayor importancia son el café, la papa, el arroz, el algodón, los espárragos, la caña de azúcar y el maíz; tanto por su uso en la dieta alimentaria interna, generación de divisas y generación de residuos para alimentación animal. Además, existen otros cultivos emergentes muy importantes, como frutas, hortalizas, legumbres, cultivos andinos y amazónicos (FAO-INIA, 2009).

La alimentación animal requiere de la utilización óptima de insumos y del conocimiento preciso de las características nutricionales. En efecto, en forrajes y subproductos agroindustriales, la variabilidad nutritiva es enorme. Por ello, es necesario caracterizar adecuadamente los insumos para la alimentación animal, según la procedencia y estado vegetativo (Maroto et al., 2011).

Una práctica común es la utilización de la papa en harina, luego del lavado, pelado e inmersión en bisulfito de sodio en concentración de 100 ppm con el objetivo de evitar el pardeamiento enzimático, luego cortar en rodajas y secarlas a una temperatura de $60{ }^{\circ} \mathrm{C}$ durante 15 horas, molerla y finalmente hacer el tamizado (Cerón et al., 2010).

Church (2010) define a los insumos como la materia prima fundamental para la alimentación animal e indica que se han clasificado más de dos mil insumos diferentes sin contar las variedades de pastos que se proporciona a los animales. Así, Córdova (1993) menciona que los insumos pueden clasificarse de acuerdo a su origen, contenido nutricional y en base a su empleo. En base a su perfil nutricional, se dividen en proteicos, los que contienen $20 \%$ o más de proteína y menos de $18 \%$ de fibra cruda en base seca; insumos fibrosos, los que poseen baja concentración de nutrientes y alto contenido de fibra, entre $18 \%$ a $35 \%$ en base seca.

El análisis proximal, o de Weende, es el esquema más empleado para describir los alimentos y comprende los contenidos de humedad, extracto etéreo, proteína cruda, cenizas, fibra cruda y extracto no nitrogenado (Nifex). Los dos últimos corresponden al total de carbohidratos (Maynard, 1989). En la región Amazonas, existe disponibilidad de insumos que podrían ser usados en alimentación animal. Existen diversas variedades de papa (Solanum tuberosa) clasificadas como de tercera, que no tiene un valor económico en el mercado, yuca (Manihot esculenta) que no fue cosechada en el momento óptimo para consumo humano, plátano (Musa paradisiaca) que debido a la distancia y carencia de vías de comunicación limitan su adecuada comercialización, la bituca o bitucón (Colocasia esculenta), tubérculo que crece de manera natural y que no es muy conocida en la alimenta- ción humana. Así mismo, existe gran diversidad de subproductos agroindustriales, que pueden ser identificados aprovechados de manera eficiente en la alimentación animal (Ravindran, 2013).

El presente estudio tuvo como objetivo caracterizar bromatológicamente veinte insumos no tradicionales disponibles en la región Amazonas, con potencialidades para ser usado en la alimentación animal.

\section{MATERIAL Y MÉTODOS \\ Lugar de estudio}

El estudio se llevó a cabo en el Instituto de Investigación de Ganadería y Biotecnología (IGBI) de la Universidad Nacional Toribio Rodríguez de Mendoza de Amazonas (UNTRM), entre los meses de marzo y septiembre de 2015. Las muestras se recogieron de zonas representativas de diferentes provincias de la región Amazonas, directamente de los predios de los agricultores muestreados según protocolos y de algunos centros de procesamiento agroindustriales (plantas procesadoras, molinos y camales).

\section{Materia prima de estudio}

Los insumos fueron arrocillo (Oryza sativa), cáscara de cacao (Theobroma cacao), pulpa de café (Coffea arabica), harina de guayaba (Psidium guajava), harina de sangre de vacuno, maíz amarillo (Zea mayz), maíz blanco (Zea mayz), pulpa de naranja (Citrus sinensis), nielen (Oryza sativa), harina de pajuro (Erythrina edulis); harina de papa (Solanum tuberosa) de diferentes variedades: aceituna, canchan, amarilis, huayro, yungay y zuela; además harina de plátano (Musa paradisiaca), polvillo de arroz (Oryza sativa), harina de bitucón (Colocasia esculenta) y harina de yuca (Manihot esculenta).

\section{Acondicionamiento de las muestras}

Las muestras procedentes de cada unidad productiva, en el laboratorio de Nutrición Animal y Bromatología de Alimentos de la UNTRM, fueron clasificadas según la variedad y distribuidas de manera sistemática. Posteriormente, y según el caso, fueron lavadas y cortadas en rodajas (papa, yuca, plátano, bitucón, cítricos y guayaba). Se sometieron a un presecado a $60{ }^{\circ} \mathrm{C}$ durante 10 a 12 horas en estufas (Ecocell, USA). Seguidamente, las muestras fueron molidas, pesadas y envasadas en bolsas de papel con sus respectivas identificaciones y finalmente se realizaron los análisis bromatológicos correspondientes, siguiendo la metodología de la Association of Official Analytical Chemists (AOAC, 2005), así se realizó el análisis proximal Van Soest para determinación de fibras y minerales. 


\section{Variables medidas, según AOAC (2005) Humedad}

Se determinó por el método de secado en una estufa al vacío a $105^{\circ} \mathrm{C}$, por un periodo de 20 a 24 horas (hasta un peso constante) (método 925.09).

\section{Proteína Cruda (PC)}

Se determinó mediante el método de Micro Kjeldahl, el cual comprende tres fases: digestión, destilación y titulación con la respectiva obtención del nitrógeno (método 984.13).

Extracto Etéreo (EE)

Se determinó por el método de extracción por hexano o método Soxhlet (método 920.39).

Fibra Cruda (FC)

Se obtuvo mediante la eliminación los carbohidratos solubles por hidrólisis, que condujo a compuestos más simples (azúcares), mediante la acción de los ácidos y álcalis débiles en caliente (método 962.09).

\section{Ceniza (CEN)}

Se determinó, mediante la eliminación de materia orgánica por calcinación a $550{ }^{\circ} \mathrm{C}$ por 3 horas (método 942.05)

\section{Extracto Libre de Nitrógeno (ELN)}

Obtenido por diferencia, al restar 100 de los resultados de proteína cruda, extracto etéreo, fibra cruda y cenizas (método 923.03) .

\section{Calcio (Ca) y Fósforo (P)}

El calcio se determinó por la técnica de precipitación como oxalato insoluble de sus soluciones amoniacales (método 927.02). El fósforo se determinó por la técnica de precipitación de fosfatos como fosfomolibdato. En el proceso, los metafosfatos o pirofosfatos se convierten a ortofosfatos por tratamiento con ácido nítrico. El precipitado se recogió, disolvió en álcali y se retituló con ácido normal. Las proteínas de origen vegetal contienen fitatos, limitando su disponibilidad del fósforo (método 965.17).

Fibra Detergente Ácido (FDA)

Se determinó el grado de digestibilidad de las fibras en el alimento. La muestra fue digerida por medio de cetil-trimetil-amonio en ácido sulfúrico y el residuo se consideró como la fibra no digerible.

\section{Fibra detergente Neutro (FDN)}

Se obtuvo mediante la separación de componentes nutricionales solubles de los que no son aprovechables o que dependen de la fermentación microbiana para su aprovechamiento. El método tiene limitaciones en su precisión cuando los valores de proteína son muy altos y fibra muy bajos.

\section{Análisis estadístico}

Para el análisis de resultados se empleó medidas de la estadística descriptiva tales como el promedio, desviación estándar, coeficiente de variación e intervalo de confianza para los promedios al 95 \%. Así mismo, se aplicó un Análisis de Varianza ajustado a un Diseño Completamente al Azar, cuando hubo significancia entre tratamientos, se realizó una prueba de promedios de HSD Tukey ( $p>0,05)$. Los datos fueron procesados y analizados mediante el programa estadístico Statistix V.8.

\section{RESULTADOS Y DISCUSIÓN}

Como resultados del análisis proximal y minerales, se muestra el valor nutricional de seis variedades de papa evaluadas y comparadas entre sí (Tabla 1). Además, en los resultados del valor nutritivo de las muestras de insumos no tradicionales en su conjunto (Tabla 2), se observan los niveles de EE, FC, CEN, ELN, PC y minerales Ca y P. Todos los valores se encuentran en porcentaje.

\section{Valor nutritivo de las variedades de papa}

No hubo diferencias estadísticas significativas para la mayoría de componentes bromatológicos comparados entre variedades de papa $(\mathrm{p}<0,05)$; sin embargo, muestran diferencia estadística para humedad y calcio, destacando las variedades zuela y amarilis; esto conllevaría a establecer un promedio general de valor nutritivo para la harina de papa (Tabla 1$)$.

Tabla 1: Comparación bromatológica entre diferentes variedades de papa

\begin{tabular}{|c|c|c|c|c|c|c|c|c|c|}
\hline Variedad & $\mathrm{n}$ & H\% & PC \% & EE \% & FC \% & ELN \% & CEN \% & Сa $\%$ & $\mathbf{P} \%$ \\
\hline Aceituna & 2 & $14,24 \pm 0,49 \mathrm{~b}$ & $8,47 \pm 0,72$ a & $0,11 \pm 0,06^{\text {a }}$ & $1,98 \pm 0,34^{\mathrm{a}}$ & $72,63 \pm 1,11$ a & $2,62 \pm 0,39^{a}$ & $0,04 \pm 0,07 \mathrm{~b}$ & $0,20 \pm 0,02^{a}$ \\
\hline Canchan & 1 & $13,09 \mathrm{c}$ & 8,63 a & $0,36^{a}$ & $2,37 \mathrm{a}$ & 76,36 a & $4,19^{\mathrm{a}}$ & $0,05 \mathrm{~b}$ & $0,21^{\mathrm{a}}$ \\
\hline Amarilis & 3 & $14,91 \pm 0,49 \mathrm{ab}$ & $9,71 \pm 0,72$ a & $0,24 \pm 0,42$ a & $3,01 \pm 0,33$ a & $69,04 \pm 1,10$ a & $3,08 \pm 0,40$ a & $0,05 \pm 0,07$ a & $0,19 \pm 0,02^{\mathrm{a}}$ \\
\hline Huayro & 11 & $14,05 \pm 0,26 \mathrm{~b}$ & $8,12 \pm 0,37$ a & $0,57 \pm 0,22$ a & $2,03 \pm 0,17$ a & $72,09 \pm 0,58$ a & $3,15 \pm 0,21^{a}$ & $0,04 \pm 0,03 \mathrm{~b}$ & $0,18 \pm 0,01^{a}$ \\
\hline Yungay & 3 & $14,06 \pm 0,49 \mathrm{~b}$ & $8,35 \pm 0,72$ a & $0,27 \pm 0,42$ a & $2,51 \pm 0,30$ a & $72,07 \pm 1,11$ a & $2,74 \pm 0,39$ a & $0,03 \pm 0,07$ b & $0,16 \pm 0,02^{a}$ \\
\hline Zuela & 10 & $15,19 \pm 0,27 \mathrm{a}$ & $7,67 \pm 0,39$ a & $0,24 \pm 0,23$ a & $2,37 \pm 0,19 \mathrm{a}$ & $71,87 \pm 0,61$ a & $2,67 \pm 0,22$ a & $0,05 \pm 0,04$ a & $0,20 \pm 0,01^{\mathrm{a}}$ \\
\hline Promedio & & 14,26 & 8,39 & 0,30 & 2,38 & 71,36 & 3,08 & 0,04 & 0,19 \\
\hline$(p<0,05)$ & & * & NS & NS & NS & NS & NS & $*$ & NS \\
\hline
\end{tabular}

Humedad: H, proteína cruda: PC, extracto etéreo: EE, fibra cruda: FC, extracto libre de nitrógeno: ELN, cenizas: CEN, calcio: Ca y fósforo: P.

NS: No significativo / $*$ Significativo: $(p>0.05)$

$\mathrm{a}, \mathrm{b}$ : Letras diferentes en la misma columna representan diferencias estadísticas, Tukey $(\mathrm{p}>0.05)$ 


\section{Materia seca}

La mayor cantidad de MS dentro de los veinte insumos no tradicionales correspondió a la cáscara de cacao con $97,12 \%$ y el menor valor para pulpa naranja con $80,32 \%$. El promedio de MS en todas las variedades de papa fue de $85,74 \%$. De ellos, destaca la variedad Canchan con $86,91 \%$ y el menor nivel la variedad Zuela con $84,81 \%$ de MS. El polvillo de arroz (89,00 \%) superó ligeramente al arrocillo y al nielen de arroz $(86,44 \%$ y 86,66 \% respectivamente), el maíz amarillo (87,03 \%) y maíz blanco $(86,75 \%)$ mostraron valores similares. Proteína cruda

Los insumos más destacados en PC fueron la harina de sangre con $78,48 \pm 0,82 \%$ que superó a la harina de pajuro y al polvillo de arroz que tuvieron $20,43 \pm 0,09 \%$ y $13,41 \pm 0,72 \%$ respectivamente. El contenido fue menor a lo hallado por Rosales y Tang (1996), quien reporta en harina de sangre de vacuno con $80,47 \%$ de PC; sin embargo, fue superior a 65,60\% obtenido por Huarcaya (1987). Mientras que el promedio de PC en las variedades de papa fue de $8,49 \%$, resultando niveles superiores al 6,40 \% hallado por Reyes et al. (2009). El contenido de PC del nielen de arroz $(8,65 \%)$ y cáscara de cacao $(8,88 \%)$, en el presente estudio, fueron menores al 11,86 \% y 10,56 \% respectivamente, reportado por Rosales y Tang (1996).

Los insumos con niveles más bajos de PC fueron la guayaba $(4,33 \%)$, harina de plátano $(3,68 \%)$, harina de yuca $(3,23 \%)$ y harina de bitucón $(5,01$ $\%)$; sin embargo, fueron superiores a lo mencionado por Reyes et al. (2009), quienes reportaron en el maíz amarillo (6,70 \%), harina de yuca $(2,10$ $\%)$ y guayaba $(0,50 \%)$, lo que indica que la calidad de dichos insumos en la región Amazonas poseen niveles de PC aceptables.

\section{Extracto etéreo}

En general, en los insumos evaluados, se encontraron bajos niveles de EE. De ellos, destaca el polvillo de arroz, maíz amarillo, harina de guayaba y maíz blanco con 14,61 \%, 3,87 \%, 3,90 \% y 3,52 $\%$ respectivamente, mientras que Reyes et al. (2009), reportaron en las harinas de yuca niveles de $(0,80 \%)$, en papa $(0,40 \%)$, en guayaba $(0,10 \%)$, maíz amarillo (3,80\%) y pajuro (0,30\%). Los insumos evaluados, indicarían y corroborarían que los vegetales no son ricos en niveles de grasas.

\section{Fibra cruda}

Los insumos con mayor nivel FC fueron la guayaba, cáscara de cacao, pulpa de café, pulpa de naranja, pajuro y polvillo de arroz con 28,41,13,39 $\%, 12,83 \%, 8,31 \%, 6,10 \%$ y $5,09 \%$ respectivamente, los insumos con alto contenido de almidón tuvieron bajos niveles de FC, entre ellos el pláta- no y el nielen de arroz alcanzaron solamente 0,90 $\%$ y $0,26 \%$ de FC. La guayaba y cáscara de cacao tuvieron diferentes niveles de fibra de los reportados por Rosales y Tang (1996). Las diferencias se deberían a las condiciones propias de las labores agrícolas, variedades y momento de cosecha.

\section{Cenizas}

Los mayores contenidos de cenizas se hallaron en la cáscara de cacao, pulpa de café y polvillo de arroz con 7,35 \%, 7,19 \% y 6,34 \% respectivamente, la papa tuvo niveles intermedios entre 2 y $4 \%$; mientras que el maíz amarillo y maíz blanco tuvieron los más bajos niveles de cenizas con 1,19 \% y 1,39 \% respectivamente. Según la Tabla Peruana de Composición de Alimentos (2009), la papa posee hasta 5, $20 \%$ de cenizas y supera al obtenido en el presente estudio y podría deberse al momento de cosecha, variedad, conservación y método de procesamiento.

\section{Extracto Libre de Nitrógeno}

Constituido por los azúcares y almidones, destacaron la yuca, plátano, arrocillo, nielen de arroz y bitucón, cuyos niveles de ELN fueron de 79,01 \%, $78,68 \%, 76,74 \%, 75,85 \%$ y $74,77 \%$ respectivamente, valores muy próximos al reportado por Rosales y Tang (1996), quienes indican que en el caso de maíz un buen contenido de ELN es de 78,79\%.

Los niveles ELN entre variedades de papas, fueron similares, en un rango del 69,04 \% a 72,63\%; mientras que la cáscara de cacao, pulpa de café, pulpa de naranja, harina de pajuro, polvillo de arroz y harina de guayaba alcanzaron menores niveles de ELN (66,96 \%, 54,65 \%, 62,59 \%, 56,33 $\%, 49,55 \%$ y $45,11 \%$ respectivamente), posiblemente por el mayor contenido de FC. Se encontró un valor mínimo de $\operatorname{ELN}(1,82 \pm 1,97 \%)$ en la harina de sangre.

En la Tabla 2, se aprecia los niveles de ELN con niveles altos y sobre todo la valorización de la energía bruta, en el cual generan un promedio de $3537 \mathrm{Kcal} / \mathrm{kg}$. Así mismo se puede apreciar los insumos que destacan en FC son los vegetales como la pulpa de café y cáscara de cacao; además la mayoría de insumos identificados son bajos en proteína no superan el 20 \% (a excepción de la harina de sangre y pajuro), los vegetales también poseen bajos niveles de grasa, calcio y fósforo, tal como se muestra en la tabla 2. 
Tabla 2. Análisis bromatológico de insumos no tradicionales de la región Amazonas

\begin{tabular}{|c|c|c|c|c|c|c|c|c|c|c|c|}
\hline Insumo alimenticio & $\begin{array}{c}\text { Humedad } \\
(\%)\end{array}$ & $\begin{array}{c}\text { Proteína } \\
(\%)\end{array}$ & $\begin{array}{c}\text { Grasa } \\
(\%)\end{array}$ & $\begin{array}{l}\text { Fibra } \\
(\%)\end{array}$ & $\begin{array}{c}\text { Ceniza } \\
(\%)\end{array}$ & $\begin{array}{l}\text { ELN } \\
(\%)\end{array}$ & $\begin{array}{c}\text { Calcio } \\
(\%)\end{array}$ & $\begin{array}{c}\text { Fósforo } \\
(\%)\end{array}$ & $\begin{array}{c}\text { FDN } \\
(\%)\end{array}$ & $\begin{array}{c}\text { FDA } \\
(\%)\end{array}$ & $\begin{array}{c}\begin{array}{c}\text { EB } \\
\text { (Kcal } / \mathrm{kg})\end{array} \\
\end{array}$ \\
\hline Arrocillo & $13,56 \pm 1,53$ & $8,30 \pm 0,22$ & $0,75 \pm 0,43$ & $0,19 \pm 0,10$ & $0,46 \pm 0,41$ & $76,74 \pm 1,25$ & $0,021 \pm 0,00$ & $0,115 \pm 0,07$ & - & - & 3558,1 \\
\hline Cacao cáscara & $2,88 \pm 00$ & $8,88 \pm 00$ & $0,54 \pm 0,00$ & $13,39 \pm 0,00$ & $7,35 \pm 0,00$ & $66,96 \pm 0,00$ & $0,16 \pm 0,00$ & $0,17 \pm 0,00$ & - & - & 3708,6 \\
\hline Café pulpa & $12,91 \pm 1,62$ & $10,77 \pm 1,01$ & $1,65 \pm 0,08$ & $12,83 \pm 0,74$ & $7,19 \pm 1,39$ & $54,65 \pm 2,55$ & $0,527 \pm 0,04$ & $0,263 \pm 0,08$ & 27,55 & 17,59 & 3387,0 \\
\hline H. de guayaba & $15,57 \pm 0,42$ & $4,33 \pm 2,34$ & $3,9 \pm 0,26$ & $28,41 \pm 0,90$ & $2,69 \pm 0,09$ & $45,11 \pm 2,66$ & $0,132 \pm 0,01$ & $0,167 \pm 0,01$ & 39,36 & 16,84 & 3507,5 \\
\hline H. de sangre & $15,04 \pm 0,50$ & $78,48 \pm 0,82$ & $0,21 \pm 0,04$ & - & $4,46 \pm 0,99$ & $1,82 \pm 1,97$ & $0,073 \pm 0,02$ & $0,13 \pm 0,01$ & 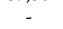 & - & 4015,7 \\
\hline Maíz Amarillo & $12,97 \pm 0,61$ & $9,67 \pm 0,68$ & $3,87 \pm 3,87$ & $1,58 \pm 0,12$ & $1,19 \pm 0,09$ & $69,94 \pm 1,98$ & $0,021 \pm 0,00$ & $0,244 \pm 0,02$ & 10,33 & 1,96 & 3691,0 \\
\hline Maíz blanco & $13,25 \pm 0,85$ & $10,01 \pm 092$ & $3,52 \pm 0,26$ & $1,85 \pm 0,34$ & $1,39 \pm 0,18$ & $69,98 \pm 1,64$ & $0,022 \pm 0,01$ & $0,277 \pm 0,04$ & 11,35 & 2,13 & 3691,3 \\
\hline Naranja pulpa & $19,68 \pm 4,71$ & $5,38 \pm 0,45$ & $1,02 \pm 0,61$ & $8,31 \pm 1,18$ & $3,03 \pm 0,28$ & $62,59 \pm 4,73$ & $0,306 \pm 0,32$ & $0,117 \pm 0,02$ & 1,10 & - & 3197,2 \\
\hline Nielen de arroz & $13,34 \pm 0,28$ & $8,65 \pm 0,38$ & $1,12 \pm 0,32$ & $0,26 \pm 0,10$ & $0,78 \pm 0,12$ & $75,85 \pm 0,22$ & $0,026 \pm 0,01$ & $0,198 \pm 0,01$ & - & - & 3579,7 \\
\hline H. de pajuro & $11,95 \pm 0,26$ & $20,43 \pm 0,09$ & $0,76 \pm 0,19$ & $6,10 \pm 1,16$ & $4,43 \pm 0,18$ & $56,33 \pm 1,33$ & $0,069 \pm 0,01$ & $0,347 \pm 0,03$ & - & - & 3585,9 \\
\hline H. de papa aceituna & $14,24 \pm 1,14$ & $8,47 \pm 2,39$ & $0,11 \pm 0,08$ & $1,94 \pm 0,07$ & $2,62 \pm 0,07$ & $72,63 \pm 3,74$ & $0,035 \pm 0,03$ & $0,20 \pm 0,02$ & - & - & 3414,3 \\
\hline H. de papa canchan & $13,09 \pm 00$ & $8,63 \pm 00$ & $0,36 \pm 0,00$ & $2,37 \pm 0,00$ & $4,19 \pm 0,00$ & $71,36 \pm 0,00$ & $0,049 \pm 0,00$ & $0,21 \pm 0,00$ & - & - & 3395,5 \\
\hline H. de papa amarilis & $14,91 \pm 0,29$ & $9,71 \pm 1,17$ & $0,24 \pm 0,16$ & $3,01 \pm 1,26$ & $3,08 \pm 0,85$ & $69,04 \pm 1,87$ & $0,046 \pm 0,02$ & $0,19 \pm 0,06$ & - & - & 3387,5 \\
\hline H. de papa huayro & $14,05 \pm 0,56$ & $8,12 \pm 0,76$ & $0,57 \pm 0,67$ & $2,03 \pm 0,24$ & $3,15 \pm 0,33$ & $72,09 \pm 1,22$ & $0,036 \pm 0,01$ & $0,182 \pm 0,02$ & 13,92 & 1,96 & 3422,5 \\
\hline H. de papa yungay & $14,06 \pm 0,85$ & $8,35 \pm 0,55$ & $0,27 \pm 0,35$ & $2,51 \pm 0,29$ & $2,74 \pm 0,35$ & $72,07 \pm 1,73$ & $0,032 \pm 0,01$ & $0,16 \pm 0,00$ & - & - & 3426,2 \\
\hline H. de papa zuela & $15,19 \pm 0,52$ & $7,67 \pm, 83$ & $0,24 \pm 0,06$ & $2,37 \pm 0,42$ & $2,67 \pm 0,49$ & $71,87 \pm 1,16$ & $0,055 \pm 0,01$ & $0,202 \pm 0,02$ & 12,40 & 2,32 & 3375,9 \\
\hline H. de plátano & $13,26 \pm 0,39$ & $3,68 \pm 0,97$ & $0,33 \pm 0,34$ & $0,9 \pm 0,17$ & $3,15 \pm 2,29$ & $78,68 \pm 2,38$ & $0,028 \pm 0,01$ & $0,123 \pm 0,03$ & 10,77 & 1,24 & 3397,7 \\
\hline H. de polvillo & $11,00 \pm 0,51$ & $13,41 \pm 0,72$ & $14,61 \pm 3,18$ & $5,09 \pm 1,31$ & $6,34 \pm 1,24$ & $49,55 \pm 5,28$ & $0,052 \pm 0,01$ & $1,217 \pm 0,09$ & 17,25 & 6,82 & 4173,0 \\
\hline H. de bitucon & $12,03 \pm 0,99$ & $5,01 \pm 1,05$ & $0,28 \pm 0,28$ & $3,26 \pm 0,39$ & $4,64 \pm 1,06$ & $74,77 \pm 1,45$ & $0,109 \pm 0,02$ & $0,227 \pm 0,02$ & 22,78 & 3,6 & 3398,1 \\
\hline H. de yuca & $12,34 \pm 0,78$ & $3,23 \pm 1,18$ & $0,44 \pm 0,14$ & $2,32 \pm 0,26$ & $2,68 \pm 0,92$ & $79,01 \pm 1,87$ & $0,109 \pm 0,02$ & $0,127 \pm 0,03$ & 5,35 & 1,18 & 3433,9 \\
\hline
\end{tabular}

FDN: fibra detergente neutro. FDA: fibra detergente ácida. EB: energía bruta (calculada en base a los componentes nutricionales de los insumos). H: harina

\section{Calcio y fósforo}

En la mayoría de los insumos analizados, el contenido de fósforo superó su respectivo nivel de calcio a excepción de la pulpa de café y pulpa de naranja (Tabla 2). Los niveles de calcio destacaron en los insumos pulpa de café $(0,52 \%)$, pulpa de naranja $(0,30 \%)$, cáscara de cacao $(0,16 \%)$ y harina de guayaba $(0,13 \%)$. El polvillo de arroz alcanzó el mayor nivel de fósforo con 1,21 \%, seguido de la harina de pajuro $(0,34 \%)$, maíz amarillo $(0,24 \%)$, maíz blanco $(0,27 \%)$ y pulpa de café $(0,34 \%)$. Los niveles de $\mathrm{P}$ en las variedades de papa fueron similares entre $0,16 \%$ y $0,20 \%$ superadas en fósforo por la harina de bituca $(0,22$ $\%)$; y los niveles más bajos fueron para el arrocillo $(0,12 \%)$, harina de sangre $(0,13 \%)$, pulpa de naranja $(0,12 \%)$, harina de plátano $(0,12 \%)$ y harina de yuca $(0,12 \%)$.

Los niveles de calcio de papa y yuca, fueron ligeramente menores a los reportados por Reyes et al. (2009); Báez et al. (1997); sin embargo, los niveles de P fueron más aceptables. Así, los niveles comparativos de Ca obtenido según Reyes et al. (2009) en la harina de yuca fue de $0,01 \%$.

Fibra detergente neutra y fibra detergente ácida El mejor perfil para FDN correspondió a la harina de guayaba $(39,36 \%)$, pulpa de café $(27,55 \%)$ y harina de bitucón $(22,78 \%)$. La harina de yuca con menor nivel (5,35\%). Los niveles de FDA más altos fueron en pulpa de café $(17,59 \%)$ y harina de guayaba $(16,84 \%)$; nivel intermedio en polvillo de arroz (6,82\%), los demás insumos alcanzaron valores menores. Se muestra una relación directa entre los niveles de FC y el contenido de FDN y FDA de los insumos.

\section{CONCLUSIONES}

En la región Amazonas, se encuentra una amplia variedad de insumos no tradicionales con buenas características nutricionales para ser incluidos en la alimentación de diferentes especies domésticas. Los principales insumos no tradicionales evaluados se pueden agrupar por su origen en frutales (cítricos, plátano, guayaba, cítricos), raíces y tubérculos (variedades de papa, yuca y bitucón), subproductos agroindustriales (pulpa de café, cáscara de cacao, arrocillo, nielen y polvillo de arroz), cereales (maíz amarillo y maíz blanco) y como fuente única de origen animal la harina de sangre.

Es posible encontrar insumos con alto contenido de proteína (harina de sangre) pero, en general, el contenido es de medio a bajo de los insumos evaluados, debido a que son productos vegetales con mayor contenido de almidones y azúcares en cantidades suficientes de energía. Además, estos aportan fibra digestible, calcio y fósforo en niveles aceptables, a excepción del extracto etéreo que fue también en general bajo en los insumos no tradicionales de la región. Solamente el polvillo de arroz destacó con 14,61 \%; esto nos permitiría aseverar que los insumos de origen vegetal aportarían energía proveniente del almidón, azucares y hemicelulosa ya que los niveles de grasas y proteína son bajos.

\section{RECOMENDACIONES}

Se recomienda continuar con la identificación, análisis y valoración nutritiva de insumos provenientes de productos, residuos agrícolas y subproductos agroindustriales, para realizar ensayos de respuesta animal con raciones con diferentes niveles de dichos insumos como fuentes potenciales de diversos nutrientes teniendo en consideración la disponibilidad y precios.

Realizar una caracterización en base a niveles de aminoácidos disponibles, micronutrientes y presencia de compuestos antinutricionales de insu- 
mos no tradicionales en estudio y así complementar los componentes evaluados.

\section{REFERENCIAS BIBLIOGRÁFICAS}

Asocciation of Official Analytical Chemists (AOAC). (2005). Official Methods of Analysis. Decim Edición. Arlington, Viginia. 22201 USA. 771 pp.

Báez, G., Pérez, A., \& Rivera, H. (1997). Obtención de Harina de Papa. Universidad Autónoma Metropolitana-Unidad Etapalapa. División de Ciencias Básicas e Ingeniería. México.

Church, D. C., Pond, W.G., \& Pond, K. R. (2010). Fundamentos de nutrición y alimentación de animales. México D.F.: Limusa S.A. 481-513

Cerón, A., Hurtado, B., Osorio, M., \& Buchely, M. (2010). Estudio de la formulación de la harina de papa de la variedad parda pastusa (Solanum tuberosum) como sustituto parcial de la harina de trigo. Biotecnología en el Sector Agropecuario y Agroindustrial. Vol 9 No. 1 (115 121) Enero-junio 2011.

Córdova, P. (1993). Alimentación animal. Lima (Perú): Editorial EDITEC.

Del Águila, A. T. (2016). Optimización de la mezcla de dietas para la elaboración de alimento balanceado con requisitos predeterminados en aves de engorde. Tesis de Maestría en ingeniería industrial. Universidad Nacional de Trujillo. Trujillo, Perú. 107 pp.

Huarcaya, H. (1987). Valor Nutricional de la harina de sangre de broilers en el trópico. Tesis Ingeniero Zootecnista. Tingo María (Perú): Universidad Nacional Agraria de la Selva. 59 pp.

Maroto, M., Gómez, C., Guerrero, G., Garrido, V., \& Pérez, M. (2011). La valoración nutricional de los alimentos para animales: Génesis de información. XXVII Curso de Especialización FEDNA. Madrid, España. 18 pp.

Maynard, L. A. (1989). Nutrición Animal. 7ma. edición. México: Editorial Mc GRAWHILL. 640 pp.

Organización de las Naciones Unidas para la Alimentación y la Agricultura (FAO) Instituto Nacional de Innovación Agraria (INIA). (2009). Informe Nacional sobre el Estado de los Recursos Fitogenéticos para la Agricultura y la Alimentación. Subdirección de Recursos Genéticos y Biotecnología. Lima, Perú. 94 pp.

Ravindran, V. (2013). Alimentos alternativos para su uso en formulaciones de alimentos para aves de corral. Revisión del desarrollo avícola $(\mathrm{FAO}), 71-73$.
Reyes, M., Gómez-Sánchez, I., Espinoza , C., Bravo, F., \& Ganoza, L. (2009). Tablas peruanas de composición de alimentos. Instituto Nacional de Salud.

Rosales, J. M. \& Tang, T. (1996). Composición química y digestibilidad de insumos alimenticios de la zona de Ucayali. Folia amazónica. Vol. 8 (2). 15 pp.

Valdivia, M. (2008). Alimentación de aves, cerdos y conejos con plátano. Universidad Autónoma de Nuevo León. Revista ACPA-1. Monterrey, México. 